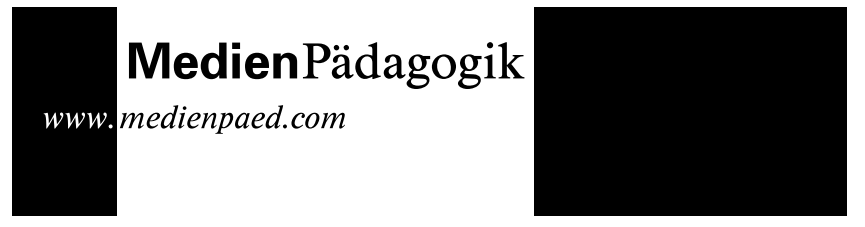

Ingeborg Schüssler

5.6 .2003

\section{Möglichkeiten des Online-Lernens - drei Beispiele aus der pädagogischen Praxis}

Die Autorin illustriert am Beispiel persönlicher Erfahrungen mit der Lernplattform WebCT Möglichkeiten des Online-Lernens. Die flexible Handhabbarkeit der Lernplattform wird anhand dreier unterschiedlicher Einsatzfelder aufgezeigt. Vorgestellt werden ein Plan- und Rollenspiel, das für die berufliche Weiterbildung konzipiert wurde, eine Veranstaltung für Lehramtsstudierende an der Universität sowie die formative Evaluation und wissenschaftliche Begleitung eines Modellprojektes.

\section{Einleitung}

Für die Formen technologiegestützten Lehrens und Lernens gibt es mittlerweile eine unüberschaubare Menge an verschiedenen Begrifflichkeiten, wie z. B. «Computer-Based-Training» (CBT), «Tele-Learning», «Virtuelles Lernen» oder auch «E-Learning» (vgl. Bloh/ Lehmann 2002, S. 17). Ohne im Einzelnen auf diese Begrifflichkeiten eingehen zu können, bezeichnet Online-Lernen im Folgenden eine Lernform, die auf der Nutzung einer Netzwerktechnologie beruht und Kommunikationsmöglichkeiten, Interventionsformen sowie den Informationsaustausch in zeitgleichen (synchronen) oder zeitversetzten (asynchronen) Arrangements über die Distanz ermöglicht.

Häufig gilt die Nutzung neuer Medien und Technologien per se als Indiz für innovative Lernarrangements und die Verbesserung der Lernergebnisse. Allerdings ergeben sich aus mediendidaktischer Sicht keine signifikanten Unterschiede zwischen traditionellen und technologiegestützten Lernformen. Wie Kerres feststellt, gibt es keine innovativen oder antiquierten Medien für Lehr-Lernzwecke, sondern «die Bedeutung eines Mediums und seine Wirksamkeit ergeben sich aus dem jeweiligen Kommunikations- zusammenhang» (Kerres 2001, S. 11). D. h. der Bildungswert bzw. die Lernwirkung des Online-Lernens lässt sich erst im Kontext einer konkreten Lernsituation erfassen (vgl. Keil-Slawik/ Selke 1998, S. 179) oder wie Lehmann und Bloh konstatieren (2002, S. 16): «Nicht die (neue) Technologie, das Medium oder der Medienverbund, sondern die Pädagogik macht den Unterschied».

Da Technologie und Medium weitestgehend verwendungsneutral sind, erschliessen sich die pädagogischen Möglichkeiten erst aus der konkreten Umsetzung. Die folgenden Beispiele zeigen, wie ein und dieselbe Technologie - in diesem Falle die Server-Software WebCT - in unterschiedlichsten Lehr-Lernkontexten, mit differenten Thematiken und Intentionen, sowie verschiedenen didaktisch-methodischen Arrangements zum Einsatz kommt.

Vorgestellt wird im ersten Beispiel ein Plan- und Rollenspiel zur Simulation einer Unternehmensplanung. Der Vorteil dieser Methoden wird vor allem darin gesehen, dass das Lernen in einem relevanten und authentischen Problemlösungskontext, d. h. unter «Ernstcharakter» stattfindet und vollständige Handlungen, d. h. von der Planung bis zur Reflexion der Ergebnisse, ermöglicht werden. Es wird gezeigt, inwieweit der Einsatz einer Lernplattform diese konventionellen handlungs- und erfahrungsorientierten Methoden unterstützen kann. Das zweite Beispiel stellt die Möglichkeiten hybrider Lehr-Lernarrangements eines Pädagogikseminars dar, wie sie sich durch die Kombination von Präsenz- und OnlineLernformen an der Hochschule realisieren lassen. Das Online-Lernen wird dabei nicht nur als «add on» zur Präsenzveranstaltung betrachtet, sondern als Möglichkeit, alternative Kommunikations- und Kooperationsformen zur Selbsterschliessung des Lernmaterials zu nutzen. Dabei werden einerseits die Selbstorganisationsgrade der Studierenden durch die zeitliche und örtliche Flexibilität des Online-Angebots erhöht und andererseits dadurch auch die Entwicklung von Medienkompetenz zukünftiger Lehrender unterstützt. Das letzte Beispiel beschreibt die Möglichkeiten des OnlineLernens im Rahmen einer wissenschaftlichen Begleitung von Modellprojekten, wie sie derzeit für einen aktuellen Projektverbund realisiert wird. Es wird aufgezeigt, wie der Entwicklungsprozess durch Datenaustausch und kontinuierliche Feedbackprozesse über eine Lernplattform so unterstützt werden kann, dass prozessorientiertes Lernen sowie formative Selbstevaluation ineinander greifen und hierdurch die Nachhaltigkeit der Projektergebnisse stärker gesichert wird. Bevor die einzelnen Praxisbeispiele 
vorgestellt werden, wird kurz der Aufbau der Lernplattform WebCT erläutert.

\section{Die Lernplattform WebCT}

WebCT ist eine Server-Software, die sowohl den Lernenden als auch den Lehrenden den Zugang zu einzelnen Lernangeboten (im folgenden Kurse genannt) über http-Technik - also mittels handelsüblicher Internet-Browser - ermöglicht (siehe 〈http://www.webct.com〉).

Das Benutzerkonzept der Software unterscheidet folgende Benutzergruppen: Systemadministrator, Kurs-Designer, Tutor und Studierende. Systemadministratoren sind für die Installation der Software verantwortlich. Sie übernehmen auch gelegentliche Wartungsaufgaben sowie unter Umständen die Verwaltung der Benutzer. Kurs-Designer und Tutoren entsprechen am ehesten einer Lehrer- oder Kursleiterrolle. Kurs-Designer haben die Möglichkeit, die Struktur von Lernangeboten festzulegen. Darüber hinaus bestimmen sie (evtl. in Abstimmung mit dem Tutor) die Inhalte von Kursen und die technischen Kommunikationshilfsmittel, die den Studierenden pro Kurs zur Verfügung stehen. Die Tutoren begleiten und moderieren den Lernprozess der Studierenden bzw. Teilnehmenden der Kurse.

Prinzipiell können alle web-tauglichen Inhalte auch in WebCT verwendet werden. Die Plattform stellt Editoren bereit, um auch Kurs-Designern mit wenig Erfahrung in web-Technik viele Gestaltungsmöglichkeiten zu eröffnen. Des weiteren sind vorgefertigte Module (sog. Tools) integriert, die Kurs-Designer in ihre Kurse einfügen können:

- Tools zur Inhaltsdarstellung und Navigation, z. B. Erstellung von Hypertext- und Hypermediainhalten, Grafikdatenbank, automatisch generierbare Inhaltsverzeichnisse und Navigationshilfen, Glossar, Volltextsuche, Index, etc.

- Tools zur Kursorganisation, z. B. Kalender, Lehrplan, Benutzeraufzeichnungen, Studierenden-Verwaltung, Registrierung von Einsendearbeiten, etc.

- (Selbst-)Evaluations-Tools, z. B. Quizzes (in Form von Befragungen), Lernfortschrittskontrollen, Bearbeitungsfortschrittsanzeigen, Tests, etc.

- Kommunikations-Tools, z. B. internes E-Mail-System, Diskussionsforen, Chat-Räume und Whiteboard

- Tools zur Personalisierung, z. B. Notizfunktion, Lesezeichen, Studierenden-Homepages, etc
Mit Hilfe dieser und anderer Tools können sehr unterschiedliche Kurse gestaltet werden, da das Arrangement aller Kursinhalte und Tools kaum systembedingten Beschränkungen unterliegt. Auch der Zugriff der Teilnehmenden auf Inhalte und Tools kann über die Studierenden-Logins durch die Kurs-Designer gesteuert werden. So können einerseits z. B. Lerngruppen gebildet werden, die jeweils nur auf bestimmte Inhalte zugreifen können und ihr jeweils eigenes Diskussionsforum besitzen. Andererseits können auch Bedingungen bestimmt werden, nach denen bestimmte Inhalte erst zu bestimmten Zeitpunkten oder erst nach erfolgreich bestandenen Tests freigegeben werden.

Die Lernplattform WebCT bietet Kurs-Designern eine grosse Anzahl an relativ leicht zu bedienenden Werkzeugen zur Erstellung und Durchführung von Online-Kursen. Hinweise oder Vorlagen zur didaktisch sinnvollen Nutzung dieser Werkzeuge in unterschiedlichen Einsatzfällen bietet das System jedoch nicht.

\section{Beispiele des Online-Lernen in der pädagogischen Praxis}

Neue Gestaltungsmöglichkeiten für Plan- und Rollenspiele

Im Gegensatz zu traditionellen Lernformen haben Plan- und Rollenspiele in der beruflichen (Weiter-)Bildung den Vorteil, dass

a. das Lernen in einem relevanten und authentischen Problemlösungskontext stattfindet

b. Grundzusammenhänge betrieblichen Geschehens verdeutlicht werden,

c. Handlungen von der Planung bis zur Reflexion der Ergebnisse ganzheitlich ausgeführt werden können,

d.die Auswirkungen eigenen Handelns durch unmittelbare Rückmeldungen vergleichsweise schnell sichtbar werden,

e. die Eigenaktivität und Selbständigkeit gefördert werden und

f. Fehlentscheidungen im Gegensatz zur Realität kein Risiko darstellen und dadurch Verhalten, Aktion und Reaktion risikofrei getestet und gelernt werden können (vgl. Fürstenau 1999, S. 13).

In bisherigen Studien haben sich die gewünschten Lernergebnisse nicht immer gezeigt, weil die Lernenden z. B. vernachlässigten, komplexe Systeme ausreichend zu explorieren, Ziele zu konkretisieren, Hypothesen zu bilden und zu überprüfen oder ihr Entscheidungsverhalten an veränderte Bedingungen anzupassen. Aus diesem Grunde werden computerbasierte Unterstützungssysteme empfohlen, weil sie erlauben, komplexe Situationen aus der Realität zu simulieren und dazu instruktionale Hilfe bereit stellen. 
$\mathrm{Zu}$ unterscheiden sind Plan- und Rollenspiele, die vor allem taktische Fähigkeiten vermitteln, wie z. B. das Pilotentraining in simulierten Cockpits, Plan- und Rollenspiele, die zum Aufbau von Wissen und Verständnis eingesetzt werden, z. B. Umweltsimulationen und schliesslich Plan- und Rollenspiele, die im Bereich strategischen Handelns verortet sind, wie das folgende Beispiel einer Fabrikplanung das unter dem Titel «FIT 2011» im Rahmen eines BLK-Projektes entwickelt wurde ${ }^{1}$ (vgl. Augustin u. a. 2000a; Augustin u. a. 2000b; Thurnes 2001). Die Wurzeln dieses Spiels liegen in einem Freeform-Planspiel zum Thema Fabrikplanung. Die Zielgruppe des Planspiels sind Studierende der Ingenieur- und Wirtschaftswissenschaften gewesen. In dieser Hinsicht richtet es sich an berufstätige Ingenieure und Wirtschaftswissenschaftler, die in ihrem Arbeitsfeld zukünftig mit verteilter Zusammenarbeit konfrontiert werden. Es geht bei diesem offenen Plan- und Rollenspiel (ohne hinterlegten Algorithmus) darum, die Teilnehmenden einen Problemlösungsprozess durchleben $\mathrm{zu}$ lassen wodurch ihnen ein realitätsnahes Erfahren der Wissens- und Entscheidungsfelder (zum Thema Fabrikplanung) ermöglicht wird. Hierzu zählt auch die Gestaltung aller Aktivitäten der Teilnehmenden aus einer realitätsnahen Rolle heraus, sowie die Interaktion mit den auch in der Realität in den Problemlösungsprozess weiteren involvierten Rollen. Ein solches Simulationsspiel erfordert die Konstruktion eines inhaltlich und zeitlich abstrahierten Ausschnittes der Realität (vgl. Portele 1977, S. 14ff.), der insbesondere im Hinblick auf die Interaktion beteiligter Personen bzw. Rollen ausgestaltet ist (vgl. van Ments 1985, S. 13). Die Gesamtkonzeption von «FIT 2011» lässt für die Teilnehmenden die fachlich inhaltliche Aufgabe eines konkreten Fabrikplanungsprojektes vordergründig erscheinen. Das Rollenspiel ist derart in das Planspiel integriert, dass die Planungsaufgabe (eher ein Planspielaspekt) nur unter Beachtung der Rollenstruktur und aufgrund der zu erlernenden Fähigkeiten und Verhalten innerhalb dieser Rollenstruktur

1 Das hier vorgestellte online Plan- und Rollenspiel «FIT 2011» (vormals MINT) wurde im Rahmen eines von der BLK «Fernstudium» geförderten Projektes am Lehrstuhls für Fertigungstechnik und Betriebsorganisation der Universität Kaiserslautern (FBK) in Zusammenarbeit mit dem Zentrum für Fernstudien und Universitäre Weiterbildung (ZFUW) sowie weiteren Partnern entwickelt. Die Autorin hat das

Plan- und Rollenspiel unter der Perspektive nachhaltigen Lernens untersucht und den Projektverantwortlichen in didaktischen Fragen beraten. gelöst werden kann (vgl. Thurnes 2001, S. 49 ff).

Einen Einblick vermittelt die folgende Darstellung der Möglichkeiten beim Aufbau eines Situations- und Rollenkonstrukts mit Hilfe der Lernplattform WebCT, die an die Ausführungen von Stammwitz und Thurnes angelehnt ist (vgl. Stammwitz/ Thurnes 2001; Thurnes 2001).

- Gestaltung des Situationskonstrukts

Die Grundlage für das Spiel bildet der gewählte situative Kontext. Hier empfiehlt sich die Ableitung und lernzielgerechte Abstraktion einer realen Situation. Hierzu zählt auch die Ermittlung der relevanten Rollen und deren Beziehungen zueinander.

Ausgehend von einer betrieblichen Situation lässt sich ein Intranet für ein Spielunternehmen innerhalb von WebCT aufbauen - also ein Kurs auf der Lernplattform, der jedoch nicht augenscheinlich als Lehr-Lernangebot fungiert, sondern wie das Intranet eines Unternehmens ausgestaltet ist.

Dieses Intranet ist für die Teilnehmenden mittels ihres Logins erreichbar. Es enthält unter anderem ein Hypertext- und Hypermedia-System, welches Dokumente zum Unternehmenskontext, zur eigenen Aufgabe, zu anderen Personen im Unternehmen, zur zu verwendenden Infrastruktur etc. bereitstellt. Ein weiterer wesentlicher Bestandteil des Intranets ist der Zugang zu unterschiedlichen Kommunikationstools - insbesondere ein unternehmensinternes E-Mail-System und Diskussionsforen.

Die Situation in FIT 2011 ist von einem realen Fall abgeleitet, der didaktisch aufbereitet wurde. Im Mittelpunkt des Spiels steht ein Unternehmen, die «Truck AG». Dieses Unternehmen besitzt Geschäftsteile und Produktionsstandorte, die weltweit verteilt sind. In einem der Produktionsstandorte werden für die kommenden Jahre Produktwechsel geplant, so dass insbesondere eine Fabrikhalle komplett neugestaltet werden muss. Da ein solches Vorhaben einerseits stark von dem Know-How und der Kreativität der Planer abhängt und andererseits Investitionssummen in mehrstelliger Millionenhöhe bedeutet, setzt die Truck AG drei Teams mit Spezialisten ein, die jeweils eine erste Grobplanung erstellen sollen. Auf Basis der geeignetsten Planung wird dann später die tatsächliche Umgestaltung der Fabrikhalle stattfinden. Die Grobplanung durch die drei Planungsteams ist der in FIT 2011 simulierte Realitätsausschnitt.

Jedes Team besteht aus sechs Teilnehmer/innen, die jeweils über Spezialkenntnisse und Wissen aus einem der Bereiche Logistik, Personal und 
Montage verfügen. Darüber hinaus sind die Mitglieder eines Teams auf drei verschiedene Orte verteilt und müssen mit Hilfe von Groupware kommunizieren, kooperieren und ihre Arbeit koordinieren. Auch alle anderen Vertreter der Truck AG sind nur auf elektronischem Wege erreichbar.

Die Aufgabe wird den Teams in Form von Briefen und Analysen seitens des Managements der Truck AG gestellt.

- Gestaltung des Rollenkonstrukts für Teilnehmende

Rollen sollten rollenspieltypisch hinsichtlich der Aspekte Funktion, Zielsetzung, Fähigkeiten und soziale Erwartungen definiert werden (vgl. van Ments 1995, S. 56-58). Innerhalb des Intranets verleiht der Login den Teilnehmenden ihre jeweilige Rollenidentität. Sie verfügen im internen EMail-System über eine entsprechende Benutzerkennung und E-MailAdresse, die sie z. B. als Geschäftsführer, Betriebsrat oder Qualitätsbeauftragten ausweist. Gegenüber der Verwendung normaler E-Mail-Adressen vereinfacht dieses Vorgehen die Identifizierung mit der Rolle und erleichtert anderen Teilnehmenden, die Rollen ihrer Mitspieler ernst zu nehmen. Auch kann die Spielleitung (selbstverständlich mit Wissen der Teilnehmenden) die Kommunikation und das Spielgeschehen besser beobachten und lenken.

Das Intranet beinhaltet des weiteren die bereits geschilderten Dokumente zur Situation in einem Hypermedia- bzw. Hypertextsystem. Der Login ins Intranet ermöglicht automatisch den Zugang zu diesen Dokumenten. Darüber hinaus eröffnet sich den Teilnehmenden durch ihren Login auch noch ein weiterer Bereich des Systems, der nicht für die anderen Teilnehmenden zugänglich ist. Dieser «private» Bereich enthält rollenspezifische Informationen (z. B. also für den Logistikplaner aus Team 1 spezielle Logistikdaten). Die technische Ausgestaltung der LernplattformSoftware erlaubt es den Teilnehmer/innen nicht, ihre jeweils privaten Bereiche den anderen Teampartnern zugänglich $\mathrm{zu}$ machen. Die Informationen in diesen «privaten» Bereichen sind weitestgehend auf die zugehörigen Rollen personalisiert. Sie bestehen - ähnlich einer Postkorbübung - aus Berichten, Analysen und Briefen, die von Mitarbeitern der Truck AG (welche Rollen der Spielleitung sind) der jeweiligen Rolle zur Verfügung gestellt werden.

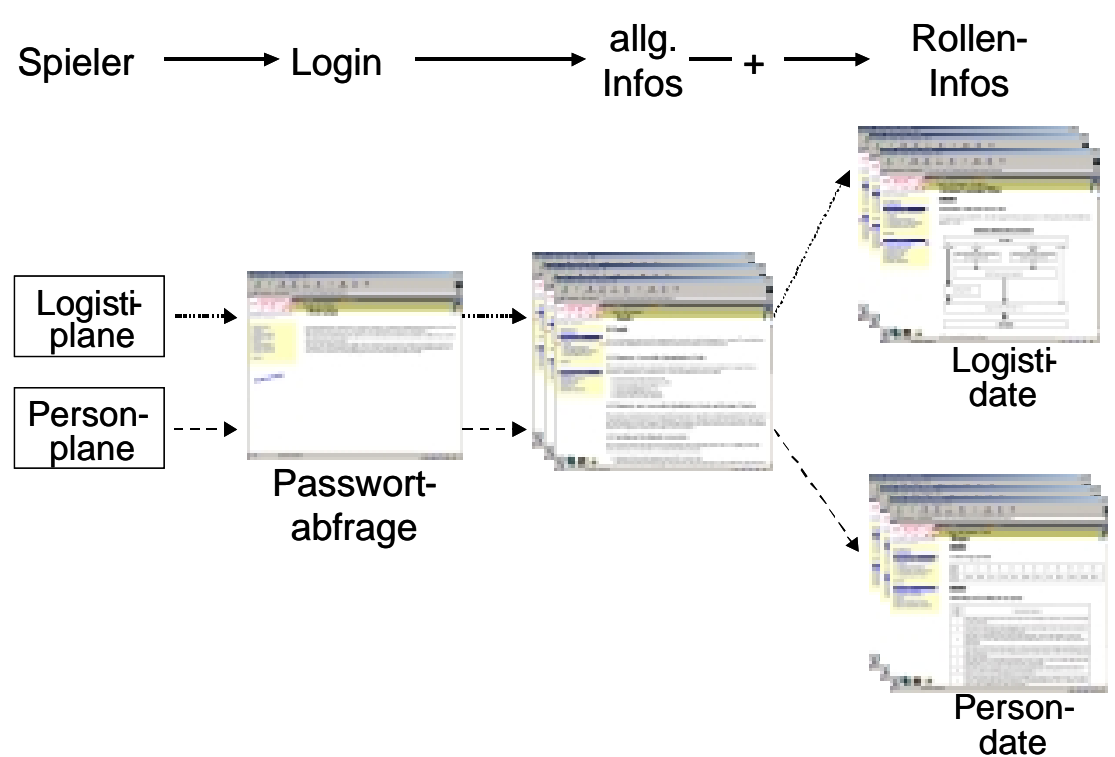

Abb.1: Rollenspezifische Informationsbereitstellung im Intranet

(Stammwitz/Thurnes 2001, S. 152)

Je nach Spiel, ist zu entscheiden, ob während dieser Einarbeitungsphase bereits die Kommunikation der Teilnehmenden untereinander ermöglicht wird. Auch die Diskussionsforen können zur weiteren Festigung der Rollen genutzt werden. Für Teams können eigene Foren erstellt werden, die nicht von anderen Teilnehmenden eingesehen werden können. Es existiert also z. B. ein Forum, zu dem alle Logistikplaner/innen automatisiert Zugang erhalten. In diesen «Communities of Practice» können sich die Spezialisten austauschen und ggf. Informationen von den Spezialisten der Konkurrenzteams im Sinne von Tauschgeschäften ver- oder einkaufen.

Das Intranet bietet auch die Nutzung eines Gruppeneditors an, mit dessen Hilfe zeitlich synchron gemeinsam an Dokumenten gearbeitet werden kann. Die synchronen Teamsitzungen werden des weiteren durch ein Konferenzsystem unterstützt, welches audiovisuell und textbasiert synchrone Kommunikation ermöglicht.

Die Gruppenarbeit bei verteilter Zusammenarbeit ist ein ständiger Wechsel von asynchroner und synchroner Kommunikation. Die Koordination der gesamten Gruppenarbeit erfordert eine stringente Planung mit Meilensteinen und Terminen für Online-Teamsitzungen. Im Intranet finden die Gruppen ein Planungssystem, welches sie bei diesen Aufgaben unterstützt. 
Neben gruppeninternen Terminen kann auch die Spielleitung dieses Planungssystem zur Steuerung des Spiels nutzen (z. B. Termine für Zwischen- und Abschlusspräsentation).

- Gestaltung des Rollenkonstrukts für die Spielleitung

Je nach Spiel besteht die Möglichkeit, die Spielleitung mittels definierter Rollen in das Spiel zu integrieren. D. h. eine oder mehrere der aus der Situationskonstruktion entstandenen Rollen werden nicht von Teilnehmenden, sondern von der Spielleitung ausgefüllt. Denkbare Vorteile hiervon sind:

- Interventionen zur Lenkung des Spiels und der Lernprozesse können aus Rollen heraus erfolgen (z. B. kann die Spielleitung in der Rolle des Geschäftsführers gezielt Arbeitsaufträge ändern). Hierdurch werden Interventionen nicht von «aussen» eingestreut, was die Realitätsnähe und Ernsthaftigkeit des Spiels gefährden könnte.

- Die Spielleitung ist in das Spiel aktiv involviert und kann die Lernprozesse aus dieser teilnehmenden Sicht beobachten und unterstützen.

Falls diese Gestaltungsspielräume vollkommen ausgeschöpft werden, sind Spiele denkbar, bei denen die Teilnehmenden nicht wissen, welche anderen Rollen von anderen Teilnehmenden oder auch von der Spielleitung eingenommen werden. Allerdings muss auch erwähnt sein, dass hohe Anforderungen an die Spielleitung gestellt sind - insbesondere, falls sie mehrere unterschiedliche Rollen verkörpern muss. Hier können unterstützend Dreh- und Rollenbücher eingesetzt werden, die ein authentisches Agieren der Spielleitung vereinfachen (vgl. Stammwitz/ Thurnes, 2001, S. 153 f).

- Grenzen und Möglichkeiten

Die dargestellte Umsetzung von Rollenspielen bzw. Plan-Rollenspielen mittels einer Lernplattform eignet sich nicht für jedes erdenkliche Spiel. In erster Linie schränkt der Zwang zur verstärkten Kommunikation mit elektronischen Medien mögliche Spielsituationen auf solche ein, in denen auch in der Realität auf diesem Wege kommuniziert wird - ansonsten wäre der Simulationscharakter des Spiels fraglich. Eine Ausnahme kann sein wenn das Kommunizieren mit elektronischen Medien selbst ein Lernziel des Spiels ist. Des weiteren müssen die hohen Anforderungen an die Spielleitung berücksichtigt werden. Bei Verwendung einer Lernplattform wie WebCT erleichtern die vorhandenen Tools und die mittels der Logins gestaltbaren rollenspezifischen Zugriffsmöglichkeiten und Gruppenbildungen die Erstellung einer glaubwürdigen Online-Spielumgebung dennoch ist der zu erbringende Gestaltungsaufwand (technisch, inhaltlich und hinsichtlich des Rollenkonstrukts) nicht unerheblich.

Für geeignete Spielsituationen bieten die oben beschriebenen Möglichkeiten jedoch grosse Potenziale. Rollenspiele oder Planspiele mit Rollenspielintegration können mit grösserem Ernstcharakter durchgeführt werden, als dies bei Präsenzspielen der Fall ist, bei denen Jeder zunächst einmal die Teilnehmende als solche wahrnimmt, die dann nur für kurze Zeit in Rollen schlüpfen. Die dargestellte Online-Variante schafft eine grössere Distanz zwischen den Teilnehmenden, da diese sich nur online in ihren Rollen begegnen. Die Spielleitung kann durch die Übernahme von Rollen Interventionen zur Lenkung des Spiels und der Lernprozesse authentisch in das Spiel einfliessen lassen. Auch lässt sich das Rollenkonstrukt über einen längeren Zeitraum aufrecht erhalten, als dies bei Präsenzrollenspielen der Fall ist. Zur Reflexion eines rein online durchgeführten Spiels kann jedoch wiederum eine Präsenzveranstaltung sehr sinnvoll sein.

Weitere Forschungs- und Entwicklungsarbeiten zur Nutzbarmachung der geschilderten Effekte zur Übertragung in den Bereich der Weiterbildung stehen noch aus.

Hybride Lehr-Lernveranstaltung im Lehramtsstudium

Im Rahmen des Projektes «Pädagogik online»² (vgl. Arnold/ Lermen 2002, Lermen 2003) werden derzeit mehrere Lehrveranstaltungen mittels der Lernplattform WebCT parallel zum Präsenzbetrieb online begleitet. Eine dieser Veranstaltungen davon ist das von der Autorin durchgefühbrte Seminar «Berufsbildungstheorie und -geschichte», welche als «hybride Lehr-Lehrnveranstaltung» im Sinne des «blended learning» aufgebaut ist (vgl. Kerres 2001, S. 3, Sauter/ Sauter 2002). Das heisst, es werden unterschiedliche methodische und mediale Formen eines Präsenz- und Online-Lernens miteinander kombiniert. Dabei versteht sich das OnlineAngebot nicht nur als additives Anhängsel der Veranstaltung, obgleich seminarbegleitende Informationen zur Verfügung stehen, wie z. B. eine «Bibliothek». Neben der im Seminar verwendeten Literatur nebst Signaturhinweisen zur schnellen Recherche in der Universitätsbibliothek stehen hier Biographien aller in der Veranstaltung auftauchenden

\footnotetext{
2 Weitere Informationen dazu finden sich auch auf den Internetseiten des Fachgebietes Pädagogik (URL: www.uni-kl.de/paedagogik) zu finden
} 
Persönlichkeiten aus der Wissenschaft mit Link-Verweisen auf Homepages oder wissenschaftliche Institute zur Verfügung. Auch der Selbstkontrollbereich mit Quizzes, Selbsttest und Fortschrittberichten sowie die Einstellung von Tafelbildern oder im Seminar erarbeiteten Diskussionsergebnissen versteht sich noch eher als Anreicherung zum traditionellen Seminarangebot, wie es mittlerweile auch vielfach an der Hochschule zum Einsatz kommt. Die Möglichkeiten der Lernplattform als solche erschliessen sich erst über die Kommunikationstools, die von den Studierenden für die Bearbeitung von Arbeitsaufträgen und Selbsterschliessungsaufgaben selbstständig genutzt werden. Für eine asynchrone Kommunikation stehen den Teilnehmenden ein internes Mailsystem und Foren zur Verfügung sowie mehrere Chat-Räume für eine synchrone Kommunikation.

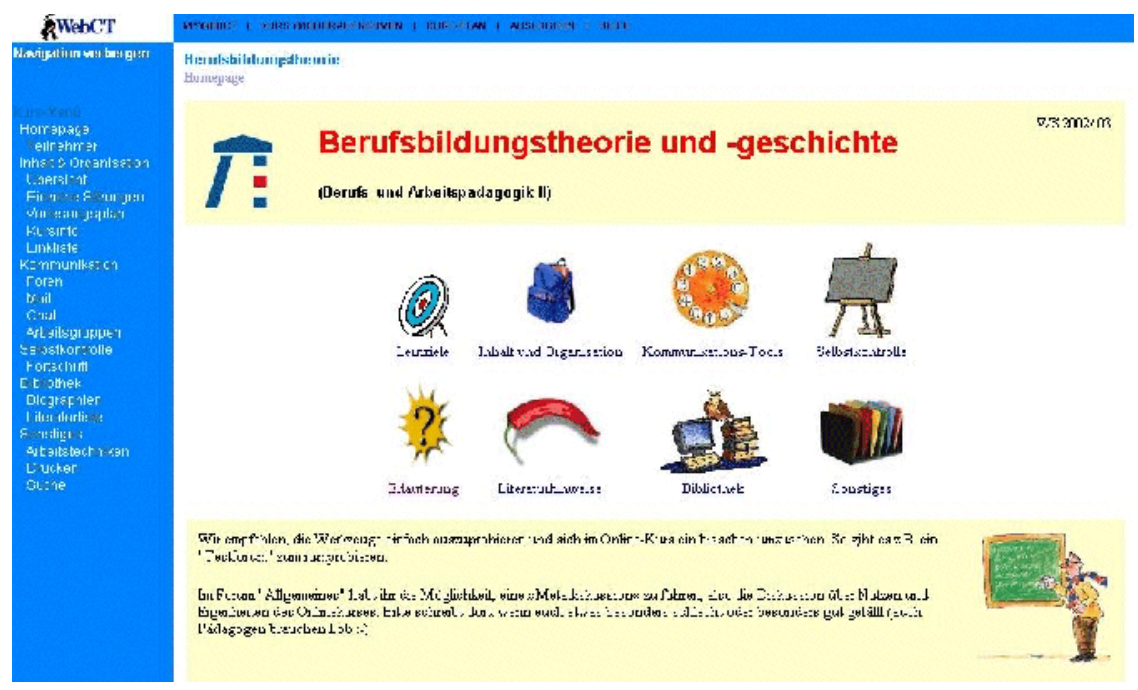

Abb.2: Eingangsportal zur Veranstaltung "Berufsbildungstheorie und-geschichte"

- Selbstorganisiertes und fachübergreifendes Lernen

Die Nutzung dieser Tools lässt sich an folgendem Beispiel illustrieren: Die Studierenden erhalten z. B. den Auftrag, die Kernthesen eines berufsbildungstheoretischen Ansatzes herauszuarbeiten und diese Erkenntnisse den Erfahrungen in der eigenen Berufsausbildung ${ }^{3}$ gegenüber zu stellen, kritisch zu diskutieren und daraus Konsequenzen für die Berufsbildungspraxis abzuleiten. Das Arbeitsergebnis, welches in Gruppen erstellt wird, soll dabei zu einem verabredeten Zeitpunkt in den Forumsbereich - für alle Seminarteilnehmenden zugänglich -eingestellt werden. Für jede Gruppe wird ein separates Forum freigeschaltet, in dem die Mitglieder sich über das Vorgehen zur Bearbeitung des Arbeitsauftrages asynchron verständigen können. Parallel dazu wird zur Halbzeit des Bearbeitungszeitraums ein Chat-Termin angeboten, in dem die Teilnehmenden sich bei Fragen direkt, d. h. synchron mit der Tutorin und auch anderen Teilnehmenden austauschen können. Die Gruppen haben nun die Möglichkeit das Forum nach ihren Bedürfnissen zu nutzen, als Marktplatz zum Austausch von Ideen und Meinungen, als Bereich, in dem die Bearbeitungsform abgestimmt, Aufgaben verteilt und/ oder Ergebnisse zur weiteren Verarbeitung eingestellt werden. Dabei müssen sich die Gruppenmitglieder selbstständig über ihre Kommunikationsformen verständigen. Zusätzlich werden die Gruppen mit einer Reihe von zusätzlichen Materialien zur Bearbeitung des Arbeitsauftrages konfrontiert, die einen selektiven Umgang damit verlangen. Das erfordert, dass sie (meist arbeitsteilig) die Materialien und angegebenen Links unter dem Aspekt der Nutzbarkeit für die zu bearbeitende Aufgabe prüfen und sich gegenseitig die Gründe ihrer Auswahl darlegen müssen.

Durch diesen Ansatz soll das Angebot nicht nur zur inhaltlichen Auseinandersetzung mit dem Seminarthema anregen und Sachkompetenz vermitteln, sondern vor allem auch im Sinne fachübergreifenden Lernens den Erwerb von Medienkompetenz ermöglichen. Denn Medienkompetenz drückt sich nicht nur in der Rezeption und Nutzung von Medien und dem Bedienen können der Kommunikations- und Informationstechnologien aus, sondern gerade auch in dem selektiven und reflexiven Umgang mit diesen Angeboten (vgl. Groeben 2002, S. 14). Es ist daher ebenso Anliegen dieser Veranstaltungskonzeption, durch die Verschränkung von Präsenz- und Online-Lernen bei den Studierenden eine solche Kompetenz schrittweise entwickeln zu helfen. Darüber hinaus ist zu prüfen, inwieweit die hier gemachten Lernerfahrungen eine Orientierung in anderen multimedialen Lernumgebungen erleichtern und den Einsatz und die reflektierte Nutzung neuer Medien auch in der zukünftigen Praxis als Berufsschullehrende

\footnotetext{
${ }^{3}$ Es handelt sich um Studierende des Lehramts an berufsbildenden Schulen, von denen alle über eine vorherige Berufsausbildung verfügen.
} 
anregen ${ }^{4}$.

- Wechsel zwischen Präsenz- und Onlinephasen

Während der Online-Phase können die Studierenden die Form ihrer Zusammenarbeit selbständig vereinbaren, was nicht ausschliesst, dass sich einzelne Gruppenmitglieder dennoch an der Universität verabreden und face-to-face über das Vorgehen verständigen, über E-Mail oder Telefon in Kontakt treten. Für Gruppenmitglieder, die diese Form nicht wählen, stehen für eine weitere direkte Kommunikation - neben den Seminarterminen - auch Chat-Rooms zur Verfügung, in denen sie sich bei Bedarf verabreden können. Die Präsenzveranstaltung dient dann dazu, die während der Bearbeitung aufgetretenen inhaltlichen Fragen der Teilnehmenden zu klären sowie solche Aspekte direkt anzusprechen, die in den Ausarbeitungen der Gruppen unklar erscheinen. Zusätzlich wird die Präsenzphase auch immer zur Reflexion der im Online-Lernen gemachten Erfahrungen genutzt. Damit soll nicht nur sicher gestellt werden, dass die Teilnehmenden sich ihrer eigenen Lernwege und -schwierigkeiten bewusst werden, sondern auch, dass sie eine unmittelbare Rückmeldung zum Aufbau und der Nutzungsfreundlichkeit der Lernplattform geben können und damit die Plattform selbst prozessbegleitend mit den Lehrenden weiterentwickeln. Das führt u. a. dazu, dass z. B. zusätzliche Wünsche nach Information oder selbst recherchierte Link-Hinweise sowie Protokolle der Präsenzveranstaltung rückwirkend zu den einzelnen Sitzungsterminen ergänzt werden. Damit erfüllt die Lernplattform gleichzeitig eine ergebnissichernde Funktion.

Damit die Studierenden die Möglichkeit haben, ihr bisheriges berufspädagogisches Wissen auch im Diskurs mit anderen anzuwenden bzw. weiter anzureichern, können sie an einem Chat-Termin mit einem Berufs-

${ }^{4}$ Die Evaluation der Veranstaltung ergab, dass $92,8 \%$ der Studierenden der Aussage zustimmen «Erfahrungen und Umgang mit Computer sind wichtige Voraussetzungen für die spätere Lehrtätigkeit». 49,9\% halten Online-Kurse für eine sinnvolle Ergänzung zum herkömmlichen Präsenzunterricht. Diese Meinung spiegelt sich auch darin, dass $57,1 \%$ der Studierenden die Verknüpfung von Präsenz- und OnlinePhasen in der hier durchgeführten Veranstaltung für sinnvoll halten. Dennoch sind 64,3\% der Ansicht, dass traditionelle Kurse reinen Online-Veranstaltungen überlegen sind. So können sich auch $85,7 \%$ der Studierenden nicht vorstellen, die Veranstaltung vollständig im Online-Modus zu absolvieren. Dieses Ergebnis spricht für die

Beibehaltung des hybriden Veranstaltungsmodus. Die vollständige Evaluation findet sich unter:

〈http://www.uni-kl.de/paedagogik/Evaluation/Evaluation_WS2002_03_BBT.pdf〉

$13 / 22$ pädagogen aus einer anderen Universität und einem Lehrenden an einer Berufsschule über neuere Konzepte in der Wissenschaft und Praxis diskutieren. Geplant ist zukünftig, die Veranstaltung in Kooperation mit einer anderen Universität sowie vollständig virtualisiert anzubieten, um dadurch auch unterschiedlichste Nutzungsformen des Online-Lernens zu erproben und zu evaluieren.

- Impulse für einen Wandel der universitären Lernkultur

Die durch das Online-Lernen ermöglichte universitäre Entgrenzung hat aber auch Auswirkungen auf das Veranstaltungsangebot. Zum einen besteht die Möglichkeit, das eigene Angebotsspektrum durch die Kooperation mit einer anderen wissenschaftlichen Einrichtung zu ergänzen. Zum anderen erfordert die Kooperation mit anderen Lehrenden den Austausch in theoretischer und methodischer Hinsicht, wodurch zugleich die fachlichen und methodischen Kompetenzen der Lehrenden erweitert und somit die Innovierung des Veranstaltungsangebots angeregt wird. Online-Lernen bietet sich dadurch selbst als Medium des wissenschaftlichen Diskurses an und kann, wenn es in diesem Sinne genutzt wird, ebenso zu einem Wandel der universitären Lernkultur beitragen (vgl. Arnold/ Schüssler 1998).

\section{Integration von Projektarbeit, Kompetenzentwicklung und (Selbst-)} Evaluation - Herausforderungen an die wissenschaftliche Begleitung Gelernt wird nicht nur in institutionellen und formellen Bildungskontexten, sondern auch in Projekten wie sie tagtäglich in Betrieben, Institutionen oder Projektverbünden stattfinden. In der Industrie wird daher versucht, diese Wissensquelle über Wissensmanagementsysteme zu erschliessen, um die darin erarbeiteten Erfahrungen auch zukünftigen Projektvorhaben zur Verfügung zu stellen. Bei den abgelegten Daten handelt sich aber häufig nur um bedeutungsarme Informationen, die durch das Fehlen von Kontextangaben und mangelnde didaktische Aufbereitung kaum sinnvoll von einem fremden Nutzer für seine Projektaufgaben zu Wissen transformiert werden können. Das in einer Organisation erarbeitete Wissenspotenzial bleibt somit häufig ungenutzt und Projekterfahrungen erlangen dadurch nur eine geringe Nachhaltigkeit. Aus diesem Grunde werden in jüngster Zeit verstärkt Versuche unternommen, Wissensmanagement und E-Learning zusammenzuführen, d. h. die zur Verfügung stehenden internen Informationen werden zusätzlich durch externe Informationen ergänzt sowie 
didaktisch und instruktional so aufbereitet, dass sie den Einzelnen bei der Konstruktion von Wissen und der Entwicklung von Kompetenzen unterstützen (Back 2001, 2002; Reinmann-Rothmeier 2002).

\section{- Mangelnde Nachhaltigkeit von Modellprojekten}

Die mangelnde Nachhaltigkeit von Projekterfahrungen stellt sich auch als Problem in bildungspolitischen oder wissenschaftlichen Modellvorhaben (vgl. Stockmann 1996). Häufig wird die Möglichkeit nicht genutzt, den Entwicklungsprozess als Lernprozess einer ganzen Organisation bzw. als Lernprozess der in die Projekte involvierten Kooperationspartner zu gestalten. In der Unterstützung dieses Entwicklungsprozesses liegt eine wesentliche Aufgabe der wissenschaftlichen Begleitung. Es geht zum einen darum, die Projekte in der Umsetzung ihres Modellvorhabens zu begleiten, z. B. durch die Unterstützung im Projektmanagement und durch die Beratung bei inhaltlichen Fragen. Zum anderen besteht die Aufgabe darin, den Lern- und Entwicklungsprozess selbst in den Projekten anzuregen und zu begleiten. Aus diesem Grunde reicht es nicht aus, die Ergebnisse solcher Vorhaben durch eine summative Evaluation zu bewerten, um daraus den Transfergehalt für die Praxis abzuleiten. Erst eine formative, prozessbegleitende Evaluation ermöglicht den Projektteams, durch kontinuierliche Feedbackschleifen, den eigenen Lernprozess sowie Problemlösungsstrategien zu reflektieren und zu analysieren sowie die Ergebnisse in das weitere Handeln zu integrieren und somit den Veränderungsprozess im Praxisfeld nachhaltig zu etablieren (vgl. Agyris/ Schön 1999). Daher muss sich die wissenschaftliche Begleitung von einem «Gutachtermodell» (vgl. Will/ Winteler/ Krapp 1987, S. 27), bei dem es lediglich um eine distanziert-objektive und quantitative Messung der Wirkungen am Ende einer Massnahme geht, zu einem so genannten «Helfer- und Beratermodell» (ebd., S. 28ff.) wandeln. Hier werden Massnahmen-Ziele, Bewertungskriterien und relevante Fragestellungen gemeinsam mit den Beteiligten herausgearbeitet und über die Kooperation der Beteiligten eine Akzeptanz der kontinuierlichen Rückkopplung (über Evaluationsbefunde) gesichert. Die Nachhaltigkeit von Modellprojekten hängt somit auch davon $\mathrm{ab}$, dass die Beteiligten evaluativ denken und handeln lernen.

- Prozessbegleitende (Selbst-)Evaluation

Die wissenschaftliche Begleitung kann diesen Selbstevaluationsprozess insofern unterstützen, als sie Methoden und Verfahren zur Verfügung stellt, die Evaluationsergebnisse für alle Projektbeteiligten aufbereitet und den Reflexionsprozess durch gezielte Impulse und Interventionen anstösst. Ziel ist es, individuelles Lernen in organisationales Lernen zu überführen, wofür Probst und Büchel (1998, S. 22ff.) drei wesentliche Bedingungen nennen:

- Transparenz, um individuelle Erfahrungen einem grösseren Personenkreis mitzuteilen und dementsprechend öffentlich zu machen,

- Integration des Wissens in neue Problemlösungen, die der Handlungsorientierung dienen,

- Kommunikation, um eine konsensuelle Verständigung über die Wirklichkeit (Zielvorstellung, Qualitätskriterien etc.) und das daraus folgende Handeln zu ermöglichen.

Diese Bedingungen lassen sich über eine Lernplattform erfolgreich implementieren, wie dies derzeit in einem konkreten Modellprojekt erprobt wird $^{5}$. So können virtuelle Kommunikationsprozesse gezielt angeregt und begleitend moderiert werden, so dass auch zwischen Präsenz-Projekttreffen ein kontinuierlicher Austausch - unter den Projekten und mit der wissenschaftlichen Begleitung - gewährleistet wird und somit unterschiedliche Projekterfahrungen transparent gemacht werden können. Dazu sind zum einen private Foren eingerichtet, die nur den Partnern eines Projektes, der wissenschaftlichen Begleitung und Projektleitung zugänglich sind sowie offene Foren, in denen aus der Projektarbeit heraus Fragen, z. B. zu Möglichkeiten der Kompetenzerfassung oder zu Formen der Projektdokumentation mit allen Projektbeteiligten diskutiert und Informationen ausgetauscht werden. Die Kommunikation über eine Lernplattform ermöglicht somit, insbesondere dann, wenn - wie im vorliegenden Falle - die zu betreuenden Projekte an verschiedenen Orten arbeiten, einen Wissenstransfer - wie in klassischen Groupware-Systemen ${ }^{6}$ - mit Hilfe des Austauschs elektronischer Daten und Dokumente. Dadurch können solche Informationen zur Verfügung gestellt werden, die für die einzelnen Projekte Handlungsrelevanz besitzen und unmittelbar für die Praxis nutzbar

5 Aktuell wird diese Form der wissenschaftlichen Begleitung von der Autorin im Projekt «Neue Lerndienstleistungen» umgesetzt. Dieses Modellprojekt wird im Rahmen des Forschungs- und Entwicklungsprogramms «Lernkultur Kompetenzentwicklung» im Bereich «Lernen in beruflichen Weiterbildungseinrichtungen» mit insgesamt vier Gestaltungsprojekten durchgeführt (vgl. 〈http://www.abwf.de/main/projekte/frame_html>; 12/2002).

${ }^{6}$ Als Groupware bezeichnet man elektronische Systeme (Hard- und/oder Software), die die Zusammenarbeit von Menschen unterstützen und aus der CSCW-(Computer Supported Cooperative Work) Forschung resultieren. 
MedienPädagogik

werden. Zum Beispiel ist es für die Projektarbeit zu Beginn notwendig, eine umfassende Kontextanalyse durchzuführen, um den Ist-Zustand der Organisation zu erheben, Ziele für die Projektarbeit zu definieren und einen umfassenden Projektplan zu erstellen. Die entsprechenden Instrumente dazu werden online zur Verfügung gestellt sowie die Projektpartner in der Umsetzung dieser Instrumente beraten.

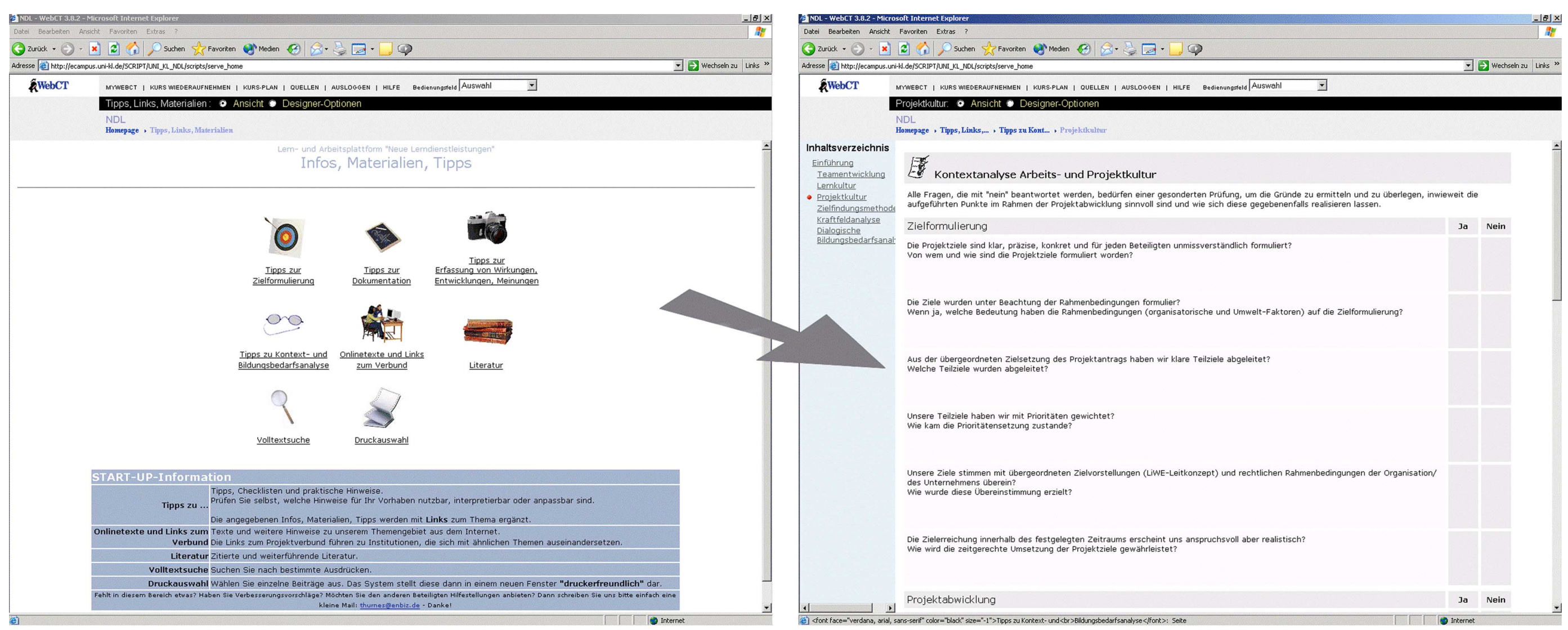

Abb.3: Arbeitsbereich «Infos, Materialien, Tipps», Beispiel: Fragebogen zur «Kontextanalyse Arbeits- und Projektkultur» 
Gleichzeitig werden die Ergebnisse in den internen Forumsbereich eingestellt, wodurch eine formative Dokumentation und Evaluation des Entwicklungsverlaufs der einzelnen Projekte möglich wird. Dadurch wird eine prozessbegleitenden Kommunikation und situations- und projektspezifische Intervention gewährleistet sowie eine kontinuierliche Rückkopplung über den Entwicklungsverlauf der Projekte sicher gestellt. Die Rolle der wissenschaftlichen Begleitung erweitert sich dann insofern, als sie möglichen inhaltlichen Input für die Modellvorhaben nicht nur organisiert und distribuiert, sondern sich auch um dessen didaktische Aufbereitung bemüht und gezielt Selbstevaluationsprozesse und daraus resultierende Handlungsmassnahmen anstösst.

Online-Lernen entwickelt sich in diesem Falle als Instrument zur Integration von Aspekten des Wissens- und Kompetenzmanagements, wodurch sich neue Potenziale für die Projektarbeit und die wissenschaftliche Begleitung eröffnen.

\section{Herausforderungen des Online-Lernens für pädagogische \\ Professionals}

Bei der Untersuchung traditioneller Lehr-Lernformen auf ihre Umsetzbarkeit in Online- oder Blended-Learning-Angebote ist deutlich geworden, dass die Interdependenz von Ziel-, Inhalts-, Methoden- und Medienentscheidungen - in Abhängigkeit zu personalen und institutionellen Bedingungen - den Rahmen für mögliche Lerneraktivitäten bilden und daher nicht eines der genannten Entscheidungsfelder, wie z. B. der Einsatz neuer Medien, losgelöst von den jeweils anderen gestaltet werden kann (vgl. Bloh/Lehmann 2002, S. 56ff.).

Die vorgestellten Beispiele verdeutlichen, dass technische Hilfsmittel wie die Lernplattform WebCT lediglich als Werkzeuge für die Ausgestaltung unterschiedlichster Lernprozesse mittels verschiedener didaktischer Inszenierungen fungieren. Das didaktische Design muss für das OnlineLernen die Potenziale dieser Werkzeuge nutzen und in Einklang mit Zielen, Inhalten, Methoden und Rahmenbedingungen bringen. Hieraus ergeben sich für die Lehrenden neue Anforderungen, welche herkömmliche Anforderungen nicht ersetzen, sondern ergänzen:

- Ein tiefergreifendes Verständnis für die technische Funktionsweise der zu nutzenden Hard- und Software ist hilfreich - jedoch nicht zwingend erforderlich. Es vereinfacht aber die Zusammenarbeit mit Programmierung und Administration und somit die technische Realisierung der eigenen Vision vom didaktischen Design.

- Die Kenntnis der Funktionalitäten (z. B. Tools bei WebCT) und ihrer Einsatzmöglichkeiten ist eine Grundvoraussetzung, um ein Online-Lernangebot entlang der entwickelten Vorstellung vom Lernprozess auszugestalten.

- Die einzusetzenden Lehr-Lernformen sollten den Lehrenden in allen Facetten bekannt sein, um kritisch prüfen zu können, inwieweit eine Umsetzung im Rahmen von Online-Lernen didaktisch sinnvoll ist, bzw. wie sich die Einsatzmöglichkeiten einschränken oder erweitern (z. B. hinsichtlich Zielgruppe oder Inhalten). Diese Kenntnisse können auch zu Überlegungen bzgl. des Einsatzes bekannter Methoden unter neuen Rahmenbedingungen (z. B. räumliche und zeitliche Verteilung der Teilnehmenden) herangezogen werden.

- Eine eher praktische Anforderung ist die, dass die Fähigkeit zur Nutzung der zu verwendenden Hilfsmittel (z. B. E-Mail-Tool, Whiteboard) bei den Lehrenden stark ausgeprägt sein sollte, damit Lernprozesse innerhalb eines gegebenenfalls gut gestalteten Online-Lernangebots nicht durch die mangelnde Partizipationsfähigkeit des Lehrenden oder Begleitenden ins Stocken geraten.

- Eine rein wirtschaftliche Kosten-Nutzen-Betrachtung ist bei OnlineLernangeboten schwieriger durchzuführen, als bei Präsenzveranstaltungen. Neben dem reinen Erstellungs- und Durchführungsaufwand treten beim Online-Lernen noch weitere Aspekte (z. B. Wiederholbarkeit, Skalierbarkeit bzgl. Teilnehmendenzahlen, Adaptierbarkeit) in den Vordergrund.

Zusammenfassend kann festgehalten werden, dass Online-Lernen als eine Erweiterung des pädagogischen Instrumentariums bezeichnet werden kann. Die einhergehende Vergrösserung des Handlungsspielraums und der Möglichkeiten geht Hand in Hand mit zusätzlichen Anforderungen, welche zum zielgerichteten Einsatz dieses Instrumentariums erfüllt sein müssen. Online-Lernen kann per se nicht die Lerneffizienz erhöhen, wohl aber neue Lernwege eröffnen.

«Letztlich ist computerunterstütztes Lernen nur bei solchen Pädagogen gut aufgehoben, die auch ohne Computer gute Pädagogen sind»(Euler 1998) 


\section{Literatur:}

Argyris, C./ Schön, D. A.: Die Lernende Organisation. Stuttgart 1999.

Arnold, R./ Lermen, M.: «Multimediales Lernen in der Erwachsenenbildung». In: PÄDForum 4/2002, S. 456-460.

Arnold, R./ Schüssler, I.: Wandel der Lernkulturen: Ideen und Bausteine für ein lebendiges Lernen. Darmstadt 1998.

Augustin, H./Kraebber, H./Thurnes, C. M.: «International problem solving using multimedia technology». In: Proceedings of the 2000 Conference for Industry and Education Collaboration, American Society for Engineering Education. Orlando, Florida 2000a, pp. 176-184.

Augustin, H./Kraebber, H. W./Smeds, R./Thurnes, C. M.: «New skills and competencies for engineering and technology: a collaborative international approach». In: Proceedings of the 2nd Global Congress on Engineering Education, UNESCO International Center for Engineering Education. Wismar 2000b, pp. 293-296.

Back, A.: «E- Learning durch Wissensmanagement bereichern: Impulse von einem umfassenden E- Learning- Verständnis für mediendidaktische Ausbildungen». In: Medien-Pädagogik. Online-Zeitschrift für Theorie und Praxis der Medienpädagogik. 2/2002 (v. 6.11.2002); 〈http://www.medienpaed.com/〉.

Back, A.: «E-Learning und Wissensmanagement zusammenführen». In: Holenstein, A./ Wilbers, K. (Hrsg.): Handbuch E-Learning. Köln 2002. Grundwerk Dezember 2001, 7.1.

Bloh, E./ Lehmann, B. (2002): «Online-Pädagogik - der dritte Weg? Präliminarien zur neuen Domäne der Online-(Lehr-)Lernnetzwerke (OLN)». In: Lehmann, B./ Bloh, E. (Hrsg.): Online-Pädagogik. Grundlagen der Berufs- und Erwachsenenbildung. Bd. 29; Baltmannsweiler 2002, S: 11-128.

Euler, D.: «Zweifelhafte Antworten auf verzweifelte Fragen ? - Möglichkeiten und Grenzen der Evaluation multimedialer und telekommunikativer Lehr-Lernarrangements». In: Schulz, M. u. a. (Hrsg.): Wege zur Ganzheit, Profilbildung einer Pädagogik für das 21. Jahrhundert. Weinheim 1998, S. 244-258.

Fürstenau, B.: «Förderung von Problemlösefähigkeit in planspielgestützten Unterricht». In: Unterrichtswissenschaft. 27 (1999), 2, S. 135-158.

Groeben, N.: «Anforderungen an die theoretische Konzeptualisierung von Medienkompetenz». In: Groeben, N./ Hurrelmann, B. (Hrsg.):
Medienkompetenz. Voraussetzungen, Dimensionen, Funktionen. Weinheim und München 2002,S. 11-24.

Keil-Slawik, R./ Selke, H.: «Forschungsstand und Forschungsperspektiven zum virtuellen Lernen von Erwachsenen». In: Baethge, M. u. a. : Kompetenzentwicklung '98. Forschungsstand und Forschungsperspektiven. Münster 1998, S. 165-208.

Kerres, M.: Mutimediale und telemediale Lernumgebungen. Konzeption und Entwicklung. 2. vollst. überarb. Aufl. München u. a. 1998.

Kerres, M.: «Online und Präsenzelemente in Lernarrangements kombinieren». In: Holenstein, A./ Wilbers, K. (Hrsg.): Handbuch E-Learning. Köln 2002, Grundwerk Dezember 2001, 4.5.

Lermen, M.: Pädagogik-Online. In: GEW-Lehrerzeitung, 1/ 2003 (im Druck).

Probst, G.J.B./ Büchel, B.S.T.: Organisationales Lernen. Wettbewerbsvorteil für die Zukunft. Wiesbaden 1998.

Portele, G.: «Zur Theorie des Simulationsspiels». In: Lehmann, J. (Hrsg.): Simulations- und Planspiele in der Schule. Bad Heilbrunn 1977. S. 9-18.

Reinmann-Rothmeier, G.: «Mediendidaktik und Wissensmanagement». In: Medien-Pädagogik. Online-Zeitschrift für Theorie und Praxis der Medienpädagogik 2/2002 (v. 30.10.2002) 〈http://www.medienpaed.com/s.

Sauter, W./ Sauter, A. M.: Blended Learning: effiziente Integration von ELearning und Präsenztraining. Neuwied 2002.

Stammwitz, G./Thurnes, C. M.: «Mit Rollen gespielt - CSCW-Techniken ermöglichen Rollen in Internetplanspielen». In: Mandl, H. u. a. (Hrsg.): Planspiele im Internet. Bielefeld 2001, S.142-156.

Stockmann, R.: Die Wirksamkeit der Entwicklungshilfe. Opladen 1996

Thurnes, C. M.: «Softskills und Planspiele - Kompetenzmanagement in Virtuellen Teams». In: Beck, U. u. a. (Hrsg.):Tagungsband edut@in 2000. Karlsruhe 2001, S. 49-59.

van Ments, M.: Rollenspiel effektiv: Ein Leitfaden für Lehrer, Erzieher, Ausbilder und Gruppenleiter. Ins Dt. übertr. u. bearb. von Katja u. Wilhelm H. Peterssen. München 1985.

Will, H./ Winteler, A./ Krapp, A.: «Von der Erfolgskontrolle zur Evaluation». In: Dies. (Hrsg.): Evaluation in der beruflichen Aus- und Weiterbildung. Heidelberg 1987, S. 11-42. 